\title{
Evaluation of advertisement existence toward attention and understanding level vehicle driver
}

\author{
Wiwik Budiawan ${ }^{1, *}$, Arfan Bachtiar ${ }^{1}$, and Veronica Desy Afriyanti ${ }^{1}$ \\ ${ }^{1}$ Industrial Engineering, Department of Industrial Engineering, 50275 Diponegoro University, \\ Indonesia
}

\begin{abstract}
When driving on the road many things can draw attention of the main task for driving, for example advertisement. The success of an advertising depends on how well it was able to communicate with the target properly. Information processing consisting of the perception process, organizing, and remembering of receiving information. This study tries to assess four-wheel drive behaviour when he saw advertisement on the road using The Eye Tracking method to know Allocation Time of Visual Spare Capacity, Attention Level and Understanding Level driver's. From 20 people who are respondents only 15 respondents have seen advertisement displayed and obtained research results that while driving, drivers have allocation of time of 6,52 second or $3,78 \%$ of the whole long drive duration. With a small percentage, the attention of driver had low results seen from the perspective of eye integration for the advertisement. In this research also known that images and colour are easier to remember compared with the number of pictures displayed. So, the cognitive recommendation that can be given are the minimum distance between advertisement of 890 meters, Maximum element in advertisement contain 8 elements, Using the dark background in light coloured or otherwise, avoid several colours that hard to read if put together and Using Uppercase or Lowercase Font Style.
\end{abstract}

\section{Introduction}

Advertisement is defined as all forms of messages about anything that delivering through media, financed by the known initiator, and addressed to part or all of the community [1]. Type of advertisement are mass media advertisement include outdoor advertisement and internet, advertorial, Built In, posters, pamphlets and running text. Due to Outdoor Advertising Association of America, outdoor advertisement divided into 4 categories, such as Billboard, street furniture, transit and alternative media. Billboard advertisement is the most choice designed to introduce a brand name. Advertisement usually located on the road with crowd traffic. Nowadays, billboard advertisement using digital technology in static one like text or picture and dynamic one like video, running text, etc. [2]

Information Processing consisting of the perception process, organizing, and remember information (e.g.: reading the advertisements on the road). The information processing

\footnotetext{
*Corresponding author: wiwikbudiawan@ft.undip.ac.id
} 
theory is about cognitive learning who describes processing, storage, and recalled back memories. On information processing, information received and stored on short-term, medium-term and long-term memory [3]. Cognitive processes are consisting of several stages such as sensation, attention, perception, think, memory and motivation.

The successful of advertising depend on how well it was able to communicate with information desire and attitude to the right person, at the right time, and at right expenses. From the information processing occurring in advertising, there is one of two things to be observed are attention levels and understanding level of advertisement target. Due to James [4], Attention defines as centralization of the mind, in the form of clear and sharp, into one of some object that simultaneous or from systematic thought .The essence of attention is vocalization, concentration and awareness. The attention is withdrawn from one thing to handle another thing effectively, and are a condition of the opposite to perplexed, daze, and unawares. While the definition of understanding is the quality of information that can be understood by consumers. The level of understanding of the individual can be increased when the information given right, clear and concise [5].

Advertising billboards on roads having target the road user as pedestrians, vehicle drivers and passengers in a vehicle. Time to read and to understand advertisement between pedestrians, the driver vehicles and passengers on it are different. Pedestrians have time to process information in advertisements longer than other because they can stop while read it. But the road users have a limited time since being in a on roads where only just glance it.

But many researchers have hypothesized that many advertisements have low involvement or not well understood by people [6]. It is because there are factors that influences people's desire to give attention in advertisements. That is factored culture as a culture the local environment and the social class; a factor social like the reference, family, and social status; a factor private age, sex, interest, the state of the economy, work, lifestyle, personality, and psychological factors as motivation, perception and trust.

Now many relevant information and research relate to allocation sight the driver in traffic, as eye tracking, visual occlusion, visual attention. etc. In many situations, driver have the time to look at targets that are not relevant for driving. This means that in a typical situation, the driver has the freedom to choose between several satisfactory information intake patterns, and there is time to glance at things that are not traffic relevancy. That visual behaviour, including the amount of "visual spare capacity", has been found to vary with driving task demand. With the visual spare capacity reserve indicate that the driver was able to doing side tasks besides whose main task to drive. In research of [7], there are two issues relating to attention while driving is targeted detection and monitor vehicle around. In research [8] estimated that a third of driver sight attention may be allocated upon an activity that is not related to the tasks driving. Based on research [9] said that driver also observes view on it non-critical like natural scenery or other things along the road. For example, in advertisements that can be pulled the attention of the main task of driving [10]. In this study, research only in static advertisement that is along the roads.

From the explanation above to be said in visual activities there are allocation of time that unconsciously done by eyes to look things that are not related to duty driving either in a vehicle and outside vehicles. One of that things that can be pulled the attention of the main task of driving is look billboards are located on the roadside. Besides pulling attention, the advertisement also must have their level of understanding whose contents can be easy to understand by advertisement target themselves. The allocation of time in his sight when doing the main task driving and also the goals of advertisement to pull attention and understanding can be used to design a research.

This study observes on four- wheeled driver behavior of a billboard advertising on the road with analyzing their level of attention and the level of understanding advertisement target, especially driver and also the best duration time that can be used advertising 
company based on available allocation time. This study uses Driving Simulator with City Car Driving Software that provides the two types of the driving environment that usually have advertisements on the road side. This study uses video data analysis to know the respondent visual process. This study aims to identify 3 aspects which is the duration time of Visual Spare Capacity, the level of attention, their level of understanding. The duration time of Visual Spare Capacity and the level of attention can be measured through eye tracking method from observation video data. For measuring the degree of understanding using questionnaire in the aftermath of the simulation. The result of data processing it may be regarded as the result of being more objective. So, that can be obtained the level of attention and the level of understanding of the driver when sees an advertisement and can recommend the best duration time that can be used by advertising company that make effective and efficient advertising.

\section{Methods}

This study is focused on the driver visual when he saw advertisement on the road. In this study, respondents will run driving simulator in the urban road conditions areas with certain route randomly. In every simulation road, there are various advertisements. The driving simulator set like real condition. The research was done in the laboratory. The simulator consists of the Logitech G27 with completeness the wheel, a pedal set, a transmission, a driver seat, CPU, sound systems, and 3 LCD TV. Software used in the simulation this is city car driving. This game is a driving simulation using approach real conditions.

1. Variables: In this research using independent variable, dependent variable and control variables. The control variables are type of driving activities, type of roads, distraction intensity, time and weather in the simulation and 7 advertisement that appear in each road. While independent variables are road contour, time distraction appears, type of road used the modern district (straight road) and the business district (many intersections). And the dependent variables are the visual spare capacity allocation time, level of attention and level of understanding the driver.

2. Research Design: From variables above, the study researchers designed to use by researchers to solve research problems. The research will be conducted by experiment within a subject that each respondent does one condition in two types of road. The simulation held in daylight and good weather with different route in every condition. Duration of one condition that have seven advertisements appear is 2-3 minutes. In the visual activity in this case, visual spare capacity time is measured by duration of driver looking away from the main task of driving until return focus on their work as seen from driving video recording. And for level of attention is measured from the point of view of ocular view. Level of understanding is measured using their level of understanding questionnaire which adjusted with research need. Into this research stage as the proportion of respondents that is used is who pass the criteria of respondents. First, respondents do an adaptation with driving simulator device. After that, respondents are obliged to wear recorder glasses in recording video mode. Respondents were required to do one condition that has a random road which on a modern district road or a business district road. Respondents had not told the purpose and objective of research for driving normally. After doing one route, respondents fill a questionnaire after research that contains four parts. The data was undertaken at random in showing each condition to avoid miss in all the condition. In addition, researcher has determined 5 routes consisting of 3 routes a modern road district and 2 routes business district.

3. Respondent: Respondents in this research are Industrial Engineering Department Diponegoro University students with the following criteria: having driving license, 
has driving experience at least one year, good physical and mental health - normal sight do not wear glasses or contact lenses and have good understanding. This research is simple experimental research simple with strict control experiment. According to [11] the size of the sample used for experimental research with small sample size between 10 and 20 samples. In this research, retrieval of sampling technique is by purposive sampling with the total sample 20 people.

\section{Result and Discussion}

Data collection consists of video data and questionnaires. Through video recording obtained the direction of movement pupil eyes be integrated into the eyes. While from questionnaire data gathered awareness of the respondents will be advertising for advertisements, elements advertising viewed by respondents and their level of understanding the respondents each advertisement.

\section{Visual Spare Capacity Allocation Time}

First, to identify Visual Spare Capacity Allocation Time is important to note whether the respondents see an advertisement or not. Less than 50 percent of the number of respondents, while for advertisement first seen much as $55 \%$ of the number of respondents. The average number of advertising ads seen by each respondent were 2.5 or means that each respondent only can see 2-3 advertising ads in one condition. From first advertisement until seventh advertising shows that the percentage of respondents that didn't see it were increasing. This occurred due to respondents were not aware of research objectives so it is more focused on driving than see an ad billboard.

To identify visual spare capacity allocation time, researchers used the questionnaire on 7 advertisement each route. In that questionnaire contains 3 parts are first part consist of question about respondent see an advertisement or not, the location and theme of the advertisement. The result of this question is right and wrong. The questionnaire could be divided into 4 categories: "See and Remember Right" category, "See and Remember Wrong" category, "See and Do Not Remember category, "Do Not See" category. Four categories were based on the human cognitive processes starting of sensation obtained from an object of vision in the advertisement. Sensation obtained then would indirectly causing attention of human beings themselves. Sensation and attention in advertising become more important because advertising is considered successful if they convey information in it to the people who has seen. In this research got that 20 respondents who perform the conditions in research, only first advertisement that has the percentage people seen more than 50 percent of advertisement onward while the percentage that does not see higher than the see. To know the visual spare capacity allocation time using data from 15 respondents who advertisement in condition. The following are shown in Figure 1 a recapitulation of the respondents' answers that is divided into four categories:

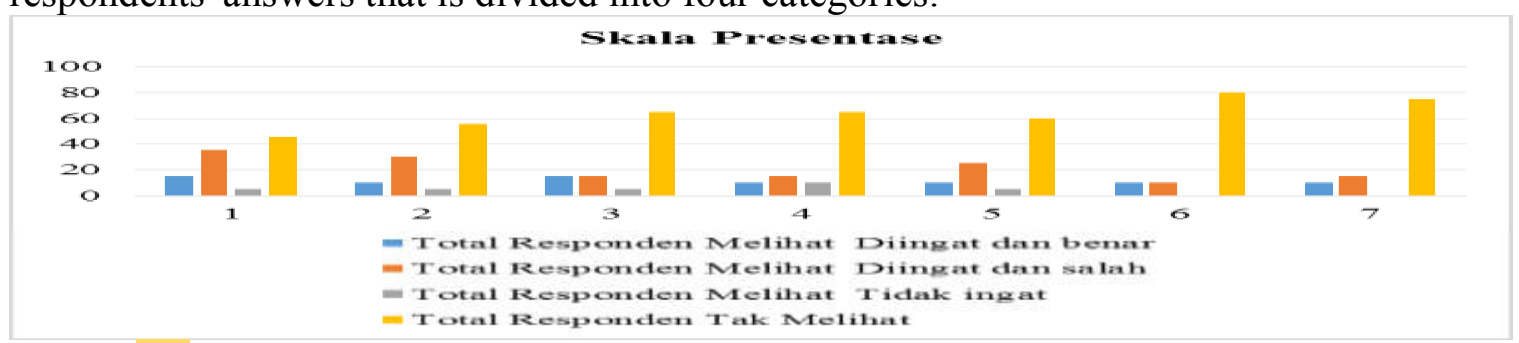

Number of Respondents See, Remember, and Answer Rightly

Number of Respondents See, Remember, and Answer Wrong

Number of Respondents See, Not Remember

Number of Respondents Not See 


\section{Fig. 1.Percentage of Respondent Awareness Chart}

Here there are significant differences of from the percentage each category. Especially for advertisement banners is not visible to drivers. To respondents who see, the percentage respondents who see and remember wrong is higher than those who see and remember right.

Based on the "seen and remember right" category got that toilet equipment advertisement having the percentage largest by $34 \%$ or 4 respondents, followed with an office rent and a flight advertisement with three respondents. This is caused toilet equipment advertisement display show detail image of toilet equipment such as a sink, a tap, a closet and type word "bathroom", so people can well aware of the ads clearly. For office rent advertisement showing a row of building and type the word "business loan", while a flight advertisement shows the images of planes and type the word "domestic flights" and "international flight" so that could deliver messages of advertisement. Visual spare capacity allocation time is the time it takes respondents when see an ad out the main task of driving. Visual spare capacity was calculated from two integration videos are video recording of Eye gaze application and video recording of recorder glasses when advertisement appear until it goes away. There is recapitulation of the following the average visual spare capacity time in each advertising of advertisement and percentage every condition of being displayed in table 1 .

Duration driving among the respondents different because of the difference in speed and average mileage each route. The distance traveled in these simulations is $1,4-3,3 \mathrm{~km}$ and average speed 40 miles/hour. In these simulations, the average distance advertisement appears in the 350 meters. Average allocation time of visual spare capacity of 6,52 seconds with standard deviations 1,548 to take one advertising billboards with the percentage comparison with driving long duration as much 3,78 \%. That can be expressed in performing a duty driving, the driver has an average allocation of time to see another one object driving in this task of billboard advertising for 6,519 seconds by standard deviations $1,548 \%$ or by $3,778 \%$ while $1,5-6$ minutes driving.

In the process see an advertisement, the visual scare capacity time longer indicates information processing longer, and oppositely. Timing is affected by advertising ads itself. For the longest time when respondents stop at traffic lights and vehicles stop. Time allocation also affected because the gap between advertisement appear every 350 meters that makes only a few more are viewed and remember correctly by respondents. Advertisement laid adjacent also affect information processing so many respondents were not remembered advertising seen.

\section{Level of Attention}

To identify attention of driver for advertisement appears can be seen from the direction of movement the eye pupil use Eye gaze application recording video who be integrated into the glasses recorder video that show visual area. Aside from the integration of two videos, the attention also be measured by the questionnaire. To make measure easy, both of video recording divided into several quadrants.

Based on the results two video recording integration and questionnaire obtained the result that of 15 respondents quadrant result of gaze direction different with the advertising quadrant ads appear. While from the 4 questions about its advertisement, shown suggests that images and color easier to remember compared with the letter and the number of images shown. As mentioned before, sensation and attention influential in communicating information in advertising. Through Eye gaze application video data to know the direction of the movement of the eye and video recording of regional sight wearing glasses recorder. A picture the results of recording video is divided into 9 quadrants to ease analysis like in Fig. 2. 

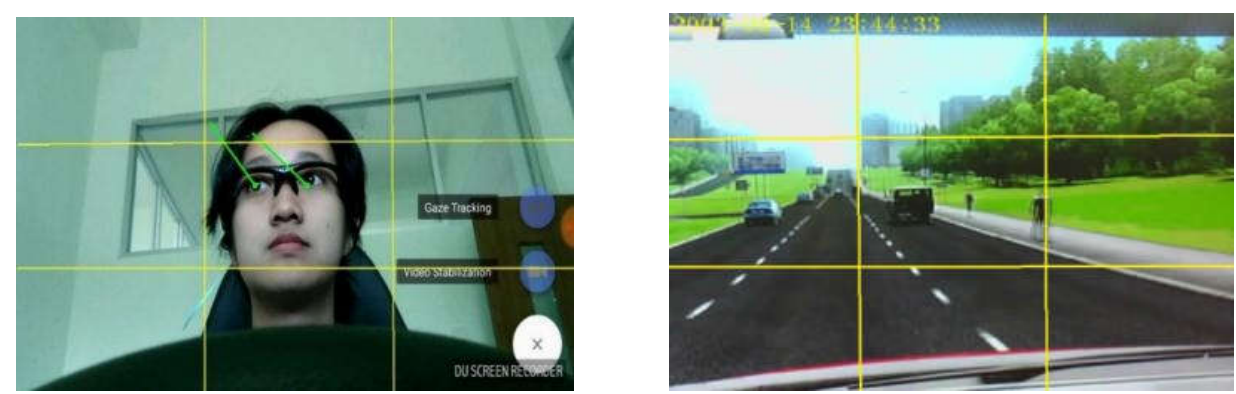

Fig.2. Quadrant of Result Integrated Video

In addition, the result of the accuracy of the quadrant in the picture compares with thirdpart of questionnaire contain of the picture, the number of images, letter and color letter that shown worth right and not right.

Than 15 respondents who see an ad ads, only 2 respondents with each see 1 st advertising with the results of the current positions of the advertising ads appear, the direction of motion of the pupil of the eye, and an answer with right. While as much as 23 different advertising are rested with the direction of the view so that not answer correctly, ad 14 are not in a quadrant From 15 respondents who see advertisement, only 2 respondents that can see an advertisement with result of advertisement appear location and eye gaze direction right. Then, 23 advertisements are located differently so respondent cannot answer correctly, 14 advertisements is not located in sight area, but respondent still can answer severe questions correctly and 13 advertisement that have a quadrant the current positions of the advertising element with a view but not answered incorrectly by respondents. At least inaccurate results the advertisement appear, the direction of movement eye pupil and answer caused respondents more focus on the main task of driving, elements in ads itself less attractive respondents' attention to see it. Based on research [12] said the driver will be realized objects in the located in the focus views or in front of vehicles. If an object outside the area, the driver see to the right and left horizontally. In addition, from the questionnaire obtained also that picture displayed and color letter easier to remember compared to the letter and the number of images shown. This suitable with research [13] that said an advertisement target spend more time to see the picture than a letter. Like in research [14] said advertising with a picture more interesting consumer attention than just a letter.

\section{Level of Understanding}

To identify the level of understanding of respondents to an advertisement seen by the duration time of the visual spare capacity and level attention respondent. In addition, to knowing the level of understanding the driver in advertisement using the fourth-part of the questionnaire. That passage consisting in four statements closed and one open statement. Four statements are relevant between images and letter that displayed, the use of images and letters that understandable easily, the use of interesting visual. To open statement, respondents answer about the meaning of advertisement are viewed. Open statement using Likert scale 1-5 (disagree; not agree; enough agree; agree; totally agree). Their level of understanding has only done in advertisement that appears in every route. This is because their level of understanding focused on the visual condition to advertisement. To identify their level of understanding, questionnaire forth-part gets the normality test. From that test got the data was not a normal distribution. From the Kruskal-Wallis test obtained from the result that there is no difference between each advertisement appeared from their level of understanding of respondents. From 4 statements are asked in the questionnaire and open statement, respondents was quite understanding about advertisement that they are seen. 


\section{Conclusion and Recommendation}

The existence of advertising ads on a road providing an effect on the driver during a main task. While driving, drivers have 6,52 second allocation time or percentage allocation of time visual spare capacity or the time to see objects unrelated to traffic of $3,78 \%$ of the whole long drive. The percentage is explaining in a duration driving, the proportion of drivers in seeing another object like advertising ads very small.

The attention when they saw advertising ads in driving visible through the direction of eye pupil movement and the eyesight area. From research getting a level of attention each person when saw advertising is low because many advertisements improper seen and improper answered by respondents. Elements advertisement shown also provides effect in attracting individual attention. In this study, picture displayed and color letter easier to remember compared to the letter and the number of the picture show.

Their level of understanding between advertisement has no significant differences that each person having a similar understandable toward advertisement who seen.

As the recommendations of the aspect of cognitive to the advertising agency who making advertising ads is the installation of advertising ads on the road more give attention at a distance more than 350 meters between advertising, so that the information distributed can be processed first. With the proportion of time very small, an advertisement should can use interesting elements advertising so can get the attention of people quickly of such as using pictures and color in advertisements. The use of language and the right word and adapted to the objective advertising can also help get attention. The use of visual spare capacity allocation time with the use of interesting and relevant elements advertisement can improve the level of understanding the driver toward advertisement. Thus, it can produce advertising billboards are effective, efficient and does not affect driving.

\section{References}

1. Anonym. Retrieved from Indonesian Advertising Company Association: http://p3i-pusat.com/definisi-iklan/ (2017)

2. M. Suyanto. World Class Advertising Design Strategy (in Bahasa). Yogyakarta: Penerbit Andi (2006)

3. R. Slavin. Educational Psychology: Theory and Practice Fourth Edition. New Jersey: Neidenheights (1994)

4. W. James. The Principles of Psychology. Vol.1. (New York: Inc, 2007)

5. G. Tsoncheva. Measuring and Accessing The Quality and Usefulness of Accounting Information. IZVESTIA - journal of University of Economics. (Varna, 2012)

6. A. Mitchell, J. Russo, \& M. Gardner. Strategy-Induced Low Involvement Perception of Advertising. Working Paper, Graduate School of Industrial Administration. (Carnegie-Mellon University, Pittsburgh, PA., 1979)

7. A. Borrowsky, D. Shinar, \& Y. Parmet. Sign Location, Sign Recognition, and Driver Expectancies. Transportation Research Part F : Traffic Psychology and Behaviour, 459-465 (2008).

8. J.F. Antin, T.A. Dingus, \& W.W. Wiewille. An Evaluation of the effectiveness and efficiency of an automobile moving-map navigational display (1990)

9. D.N. Lee, \& M.F. Land. Where we look when we steer. Nature, 742-744. (1994) 
10. J. Equist, T. Horberry, \& I. Jhonston. Effect of advertising billboard during simulated driving. Applied Ergonomics, 619-626 (2011)

11. Roscoe. Business Research Methods (in Bahasa). (Jakarta: Salemba Empat, 2006)

12. M. Crundall, E. Van Loon, \& G. Underwood. Attraction and distraction of attention with roadside advertisement. Accident Analysis and Prevention, 671-677 (2006)

13. Q. Li, Z. Huang, \& K. Christianson. Visual Attention toward Tourism Photographs with text: An Eye Tracking Study. Tourism Management, 243-258 (2016)

14. R. Pieters, \& M. Wedel. Attention capture and transfer in advertising: Brand,Pictorial and Text Size Effect. Journal of Marketing, 36-50 (2011) 